\title{
BMJ Open Efficacy of initial haemopurification strategy for acute paraquat poisoning in adults: study protocol for a randomised controlled trial (HeSAPP)
}

\author{
Jian-Wei Cui, ${ }^{1}$ Yinyan Xu, ${ }^{2}$ Yibo Wang, ${ }^{3}$ Yan-Xia Gao, ${ }^{3}$ Shigong Guo, ${ }^{4}$ Meng Wang, ${ }^{5}$ \\ Xin Lu, ${ }^{1}$ Shiyuan Yu, ${ }^{1}$ Yong Ma, ${ }^{1}$ Ding Yuan, ${ }^{3}$ Lu Che, ${ }^{3}$ Pei Sun, ${ }^{3}$ Xuezhong Yu, ${ }^{1}$ \\ Huadong Zhu, ${ }^{1} \mathrm{Yi} \mathrm{Li}^{1}$
}

To cite: Cui J-W, Xu Y, Wang Y, et al. Efficacy of initial haemopurification strategy for acute paraquat poisoning in adults: study protocol for a randomised controlled trial (HeSAPP). BMJ Open 2018;8:e021964. doi:10.1136/ bmjopen-2018-021964

- Prepublication history for this paper is available online. To view these files, please visit the journal online (http://dx.doi. org/10.1136/bmjopen-2018021964).

Y-XG and YL contributed equally.

Received 29 January 2018 Revised 12 April 2018 Accepted 18 April 2018

\section{Check for updates}

${ }^{1}$ Emergency Department, Peking Union Medical College Hospital, Beijing, China

${ }^{2}$ Department of Neurology, Peking Union Medical College Hospital, Beijing, China ${ }^{3}$ Emergency department, The First Affiliated Hospital of Zhengzhou University, Zhengzhou, China

${ }^{4} 0 x f o r d$ Centre for Enablement Nuffield Orthopaedic Centre, Oxford, UK

${ }^{5}$ Department of Science and Technology, Beijing Tiantan Hospital, Capital Medical University, Beijing, China

Correspondence to Dr Yan-Xia Gao; gaoyanxiazzu@163.com and Dr Yi Li;

billiyi@126.com

\section{ABSTRACT}

Introduction Paraquat $(P Q)$ is a widely used herbicide which is inexpensive and easily accessible for people in rural areas. A small amount of $P Q$ ingestion could be lethal, yet currently, the optimal treatment is still controversial. Extracorporeal therapies (ECTR) have been practised in $\mathrm{PQ}$ poisoning management, though limited evidence could be obtained to suggest its superiority over conservative therapy. Haemodialysis (HD) and haemoperfusion (HP) are most commonly used, while some institutions also choose HP-HD concurrent therapy. The object of the present trial is to investigate whether haemopurification therapy can reduce mortality compared with conservative

therapy.

Methods and analysis This is a planned single-centre, non-blinded, randomised controlled trial. Acute $P Q$ poisoned adults who have orally ingested $P Q$ within 24 hours would be recruited. A total of 360 patients would be recruited and randomly assigned to four groups, that is, $\mathrm{HP}, \mathrm{HD}$, concurrent HP-HD and control, at a 1:1:1:1 ratio. Subjects would be also stratified by their urine dithionite test results. Primary outcome is 28-day all-cause mortality. Secondary outcomes include survival time, all-cause mortality at the 3rd, 7th and 60th day, rate of major complications, Acute Physiologic and Chronic Health Evaluation score and Poisoning Severity Score, etc. Ethics and dissemination The protocol and informed consent documents have been approved by the Ethics Committee of The First Affiliated Hospital of Zhengzhou University in September 2017 (approval number: 2017$\mathrm{KY}-10$ ). The result of this trial would be submitted to peerreviewed journal.

Trial registration number NCT03314909; Pre-results.

\section{INTRODUCTION}

Among 1.6million violent deaths every year in the world, half are suicidal and $63 \%$ of these occur in the Asia-Pacific region. ${ }^{1}$ Pesticide suicide accounts for up to one-third of all suicides worldwide every year. ${ }^{2}$ Being inexpensive and easily accessible, paraquat (PQ), a water-soluble toxic organic herbicide
Strengths and limitations of this study

- This is the first parallel randomised controlled trial to compare the efficacy of haemoperfusion (HP), haemodialysis (HD), concurrent HP-HD and non-haemopurification treatment in acute paraquat $(\mathrm{PQ})$ poisoning.

- Patients will be stratified by the result of urine dithionite test.

- The primary outcome is 28-day mortality.

- Subgroup analysis based on time lapse from PQ ingestion to treatment may provide reference for initiation time of haemopurification.

- The limitation of this study is the unavailability of serum $\mathrm{PQ}$ concentration.

(1,1-dimethyl-4,4-bipyridine cationic salt) is still widely consumed in some countries like China, and occasionally serves as poison. ${ }^{3}$

A very small amount of PQ can cause death in human. A study of 375 participants reports that patients with a plasma PQ concentration higher than $3.44 \mu \mathrm{g} / \mathrm{mL}$ died, ${ }^{4}$ though some other studies indicate a relative higher upper limit for survivors. ${ }^{56}$ The mortality of $\mathrm{PQ}$ is remarkably high (ranging from $42.7 \%$ to $90 \%^{7-9}$ ), but unfortunately, there is still no effective treatment for confirmed $\mathrm{PQ}$ poisoning. The main mechanism of $\mathrm{PQ}$ intoxication is generation of free radicals and oxidative stress, and some studies claim that immunosuppressive therapy can improve survival rate. ${ }^{9} 10$ Considering the physical characteristics of $\mathrm{PQ}$, for example, relatively low volume of distribution (1.2-1.6L/ $\mathrm{kg}),{ }^{3}$ low molecular weight and low protein binding rate, it is reasonable to propose that extracorporeal theraphies (ECTR) may benefit patients with $\mathrm{PQ}$ poisoning.

Haemodialysis (HD) purifies blood by filtering poisonous molecules through a 
selectively permeable membrane, especially molecules with a small molecular weight and low protein binding rate. It can also correct acid-base disturbance in patients. Theoretically, HD should be the ideal treatment for acute PQ poisoning in view of its physical characteristics. However, HD is not widely applied in practice, and the Expert Consensus on Acute PQ Poisoning in China recommends HD as a supplementary therapy for patients complicated with acid-base disturbance. ${ }^{11}$ Little evidence could be obtained in $\mathrm{HD}$ for $\mathrm{PQ}$ poisoning treatment in the last 30 years. In an experimental model, it is demonstrated that after $90 \mathrm{~min}$ of $\mathrm{HD}, \mathrm{PQ}$ clearance remains static in vitro $(179 \mathrm{~mL} / \mathrm{min}) .{ }^{12}$ Compared with the high apparent renal clearance of $\mathrm{PQ}(1.17 \mathrm{~L} /$ hour $)$ in vivo, ${ }^{13}$ HD seems to have a limited effect on PQ clearance, probably due to the limitation of HD filter material. With the improvement in filter, HD has a twofold increase in small molecule clearance compared with 40 years ago ${ }^{14}$; thus, further research is needed to evaluate the treatment effect of HD in PQ poisoning management.

Haemoperfusion (HP) removes blood toxicants by absorbing them through a column and is another choice for $P Q$ poisoning treatment. As it has a superior PQ clearance over HD in vivo, ${ }^{12}$ it has become the standard treatment for PQ intoxication in many countries. ${ }^{11}{ }^{15}$ Several retrospective studies report that HP could significantly improve $\mathrm{PQ}$ plasma clearance and reduce mortality compared with control groups, ${ }^{16}{ }^{17}$ while other studies point out that patients would benefit from HP only when it is administered early from the onset of poisoning. ${ }^{12} 18$ In one prospective clinical trial, Li et al reports that HP could enhance PQ clearance, but no conclusion was drawn on mortality. ${ }^{20}$ In addition, the toxicokinetics of $\mathrm{PQ}$ during HP are poorly understood. Although some evidence from China suggests that HP and HD concurrent therapy (HP-HD) can significantly reduce mortality, ${ }^{21-25}$ it is not a standard therapy in $P Q$ poisoning. High costs and long therapeutic duration may have hindered its application in clinical practice.

The hypothesis of the present study is that early haemopurification therapies may reduce mortality in acute PQ poisoned patients. This is a single-centre, parallel, non-blinded randomised controlled trial to investigate the superiority of HD, HP and HP-HD concurrent therapy compared with conservative therapy during acute PQ poisoning. Allocation ratio of each group is 1:1:1:1.

\section{METHODS AND ANALYSIS \\ Study setting}

Patient recruitment would be completed in The First Affiliated Hospital of Zhengzhou University, a comprehensive tertiary medical centre in Henan Province, China, with 50 beds in emergency intensive care units (EICU). The estimated number of admitted acute PQ poisoned patients ranges from 50 to 200 persons per year. To assist participant enrolment, after acceptance of this protocol, a notice of this trial would be sent to the emergency room (ER) of all secondary hospitals in Henan Province to improve transference to the First Affiliated Hospital of Zhengzhou University. Considering the fact that intervention would be administered in ER setting, and the relatively short duration of assigned haemopurification, adherence of patients is promising. Patients' families would receive full explanation of treatment plan and continuous follow-up in order to promote adherence.

\section{Study population}

On admission to ER, patients suspected with PQ intoxication would receive a urine dithionite test, and only those with a positive result would be invited to participate in this trial. The urine dithionite test would be measured by Spectrophotometer Type 721, and the minimal measurable concentration of $\mathrm{PQ}$ is $0.2 \mu \mathrm{g} / \mathrm{mL}$. Detailed inclusion and exclusion criteria are listed as follows.

\section{Inclusion criteria}

Patients meeting with all of the following criteria would be included in this trial: (1) Suspected PQ ingestion history (intended or accidental), which is confirmed by positive urine dithionite result (light blue, navy blue and dark blue). (2) Arriving at the ER within 24 hours after PQ digestion. (3) Age: 18-70 years old. (4) No known current pregnancy or lactation. (4) Absence of cardiac arrest after poisoning, and no previous or present history of chronic kidney disease (CKD), chronic liver disease, respiratory failure, chronic obstructive pulmonary disease (COPD), asthma, heart failure, pancreatic disease, acute coronary syndrome (ACS) or stoke. (5) No known combined ingestion with other poisons or alcohol. (6) No previous blood purification treatment prior to admission. (8) No known participation in other medical trials. (9) Agreement on informed consent.

\section{Exclusion criteria}

Patients in any one of the following conditions would be excluded: (1) Patients who are unable to comply with the procedures of the present trial, including those who change therapy or withdraw treatment. (2) Patients who develop severe allergic response to HP materials. (3) Patients who do not receive intervention within 4 hours after admission in reality.

\section{Allocation randomisation and concealment}

All participants would be randomly stratified into three blocks according to the result of urine dithionite test, that is, light blue, navy blue and dark blue. Block length is set at 12. With the help of SAS V.9.3, patients in different blocks would be allocated to four groups, namely the HD group, HP group, concurrent HP-HD group and conservative therapy group (control group), at a 1:1:1:1 ratio (figure 1).

Due to the apparently different equipment of the interventions, it would be impractical to blind the present trial; therefore, both patients and physicians would be aware of the exact treatment that the patients would receive. A sealed envelope with the allocation 


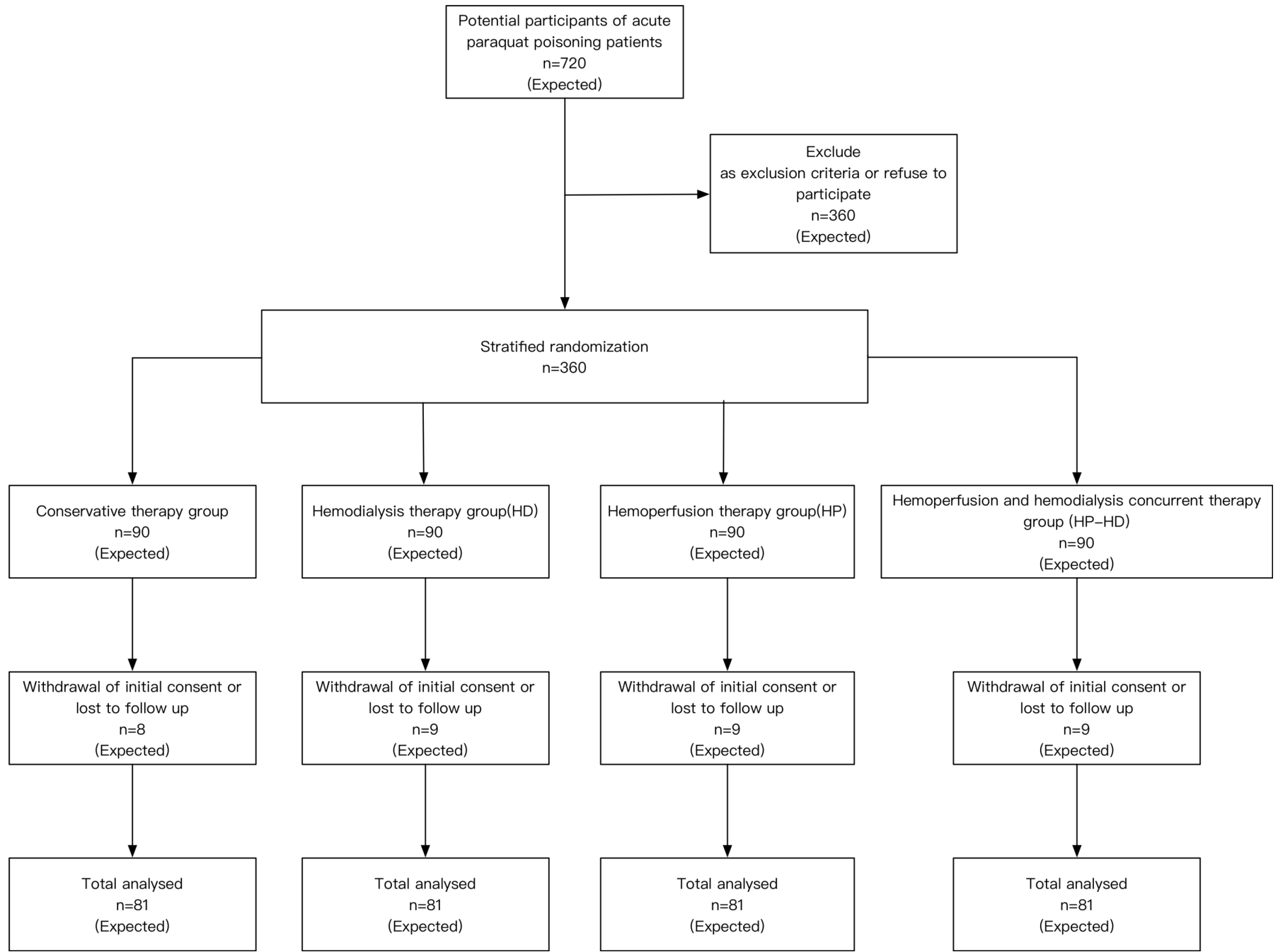

Figure 1 Diagram of the protocol (planned).

information would then be sent to the physician in charge of the patient after stratified grouping. To reduce assessor bias, blood samples and chest radiograph would be collected and examined by staff independent of this study.

\section{Intervention}

The intervention under investigation includes conservative therapy, HP alone, HD alone, and HP-HD concurrent therapy under the Guideline of Chinese Blood Purification for Acute Paraquat Poisoning Patients. ${ }^{26}$

\section{Study procedure}

Physicians involved in the study would receive standardised training in carrying out this trial. On enrolment, informed consent, basic demographic information and collateral history would be taken from the patients or their next of kin (table 1). PQ ingestion volume would be estimated as follows: 1 mouthful of liquid for women $=22 \mathrm{~mL}$ and one mouthful for men=28 mL. ${ }^{27} \mathrm{PQ}$ ingestion amount, defined as $\mathrm{PQ}$ concentration $\times \mathrm{PQ}$ ingestion volume, would be calculated. Physicians would also assess the

Table 1 The form of basic demographic information and collateral history

Time of $\mathbf{P Q}$

ingestion ingestion

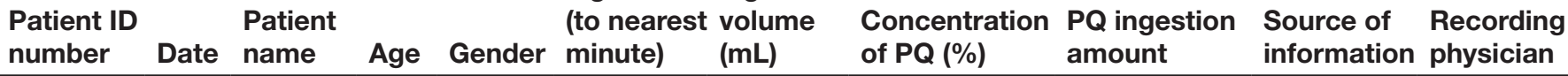

$\mathrm{PQ}$, paraquat. 


\section{Table 2 The form of initial assessment}

Patient ID number

\begin{tabular}{l} 
Date \\
Group \\
Time to intervention \\
Urine test result \\
Complete blood count \\
BMI \\
Smoking history \\
Alcohol history \\
Blood gas analysis result \\
Liver function \\
Pancreatic function \\
Kidney function \\
\hline Blood lactic Acid \\
\hline Diabetes history \\
\hline Hypertension history \\
\hline APACHE II score \\
\hline Poisoning Severity Score (PSS) \\
\hline
\end{tabular}

APACHE II, Acute Physiologic and Chronic Health Evaluation; BMI, body mass index.

participants by various scores (table 2), including Acute Physiologic and Chronic Health Evaluation (APACHE II) score and Poisoning Severity Score (PSS).

On suspected diagnosis of $\mathrm{PQ}$ poisoning, all patients would receive gastric lavage with room warm water $(\geq 5 \mathrm{~L})$ and $1 \mathrm{~g} / \mathrm{kg}$ active charcoal via nasogastric tube. After confirmed diagnosis by urine dithionite test, intervention would be initiated on acquisition of informed consent and randomised allocation, which would take less 1 hour after admission ideally. Subsequent treatment varies by groups:

1. HD group: participants would receive 4 hours of HD therapy a day for three consecutive days.

2. HP group: participants would receive 4 hours of HP a day for three consecutive days.

3. HP-HD group: participants in this group would receive 4 hours of HP HD concurrent therapy for consecutively 3 days.

4. Control group: participants in this group would receive conservative treatment (see below).

According to the Chinese Guideline on Management of Paraquat Poisoning, ${ }^{11}$ all patients groups would receive standard treatment as follows. Methylprednisolone $15 \mathrm{mg} / \mathrm{kg}$ /day together with cyclophosphamide $15 \mathrm{mg}$ / $\mathrm{kg} /$ day would be administered for the first week. After the first week, methylprednisolone would be reduced by $40 \mathrm{mg}$ every 3 days, while no more cyclophosphamide would be given. Patients would be given supplemental oxygen only if their $\mathrm{PaO}_{2}$ falls below $40 \mathrm{~mm} \mathrm{Hg}$ or in case of acute respiratory distress syndrome (ARDS).
Procedure of HD

1. Preparation: Place a dual-lumen catheter in the internal jugular vein, or place a dual-lumen catheter in the femoral vein if needed. Equip the HD machine (Fresenius 4008s. Cartridge: Fresenius Fx60. Both by Fresenius Medical Care AG Co, Germany). Rinse the pipeline with $1 \mathrm{~L}$ of normal saline (NS) at a speed of $100 \mathrm{~mL} / \mathrm{min}$. Set the volume of dialysis at $300 \mathrm{~mL}$, and run the dialysis machine in close loop for $10 \mathrm{~min}$.

2. Anticoagulation: Inject $60-80 \mathrm{IU} / \mathrm{kg}$ low-molecular-weight heparin (LMWH) 20-30 min before HD.

3. Therapy and surveillance: connect the pipeline to the catheter, and run the dialysis machine at a speed $(\mathrm{mL} / \mathrm{min})$ four times as the patient's weight $(\mathrm{kg})$. Dialysis solution speed should be set at $500 \mathrm{~mL} / \mathrm{min}$. Run HD for 4 hours; meanwhile, closely monitor the patients' vital signs. During HD, anticoagulation function should be monitored by transmembrane pressure (TMP) of dialyser. If TMP $>250 \mathrm{~mm} \mathrm{Hg}$, additional LMWH should be added.

\section{Procedure of HP}

1. Preparation: Establish a dual-lumen catheter in the internal jugular vein, or in the femoral vein if needed. Equip the HP machine (Jafron model JF-800. Cartridge: HA330. Both by Jafron Biomedical Co). Rinse the whole pipeline with $5 \%$ glucose solution at a speed of $100 \mathrm{~mL} /$ min until the pipeline is filled with glucose solution. Then rinse pipeline with NS at a speed of $200 \mathrm{~mL} / \mathrm{min}$. The total volume used for rinsing is $2000 \mathrm{~mL}$.

2. Anticoagulation: Rinse the pipeline with $500 \mathrm{~mL}$ NS mixed with $4 \mathrm{mg} / \mathrm{dL}$ heparin. Ten minutes later, rinse the pipeline with $300 \mathrm{~mL}$ NS. Connect the pipeline to the catheter on the patient. Inject $0.5-1.0 \mathrm{mg} / \mathrm{kg}$ heparin, then add heparin at a speed of $10-20 \mathrm{mg} /$ hour based on coagulation status (keep activated partial thromboplastin time (APTT) 50\% above upper limit of normal). Stop adding heparin $30 \mathrm{~min}$ before the end of each course.

3. Surveillance: Run HP for 4 hours a day. Monitor vital signs during HP and prevent hypotension. Optimal flow velocity of extracorporeal blood flow ranges from 100 to $200 \mathrm{~mL} / \mathrm{min}$. Change the HP cartridge as soon as any charcoal appears in the blood flow.

\section{Procedure of HP-HD}

1. Preparation: Place a dual-lumen catheter in the internal jugular vein, or in the femoral vein if needed in the ER. Equip the blood purification machines (HP and HD machines and cartridges as mentioned above). The outlet of the HP cartridge should be connected with the inlet of HD machine. Rinse HP pipeline and HD pipeline with $1 \mathrm{~L}$ of NS mixed with $3000 \mathrm{IU}$ heparin at a speed of $100-150 \mathrm{~mL} / \mathrm{min}$, followed by $600 \mathrm{~mL}$ of NS containing $3000 \mathrm{IU}$ heparin.

2. Anticoagulation: Inject LMWH 50-60 IU/ kg as loading dose, then maintain at a speed of $400 \mathrm{IU} / \mathrm{h}$ and adjust dose according to TMP (keep TMP $\leq 250 \mathrm{~mm} \mathrm{Hg}$ ). 
3. Run HP-HD: Connect the inlet of the HP cartridge to the catheter, and run the machine for 4 hours. Blood flow speed ranges from 100 to $200 \mathrm{~mL} / \mathrm{min}$. Dialysis solution speed is $500 \mathrm{~mL} / \mathrm{min}$. HP cartridge should be changed as soon as any charcoal appears. Patients' virtual signs should be monitored during treatment.

\section{Sample size and study power}

The hypothesis of the present trial is that all of the active arms, that is, HP, HD and HP-HD concurrent therapy, have a lower mortality compared with conservative therapy in $P Q$ poisoning treatment. Based on this assumption, we searched on several databases, that is, PubMed, EMBASE, SCI, Wanfang Data and CNKI, and found no research had compared HP, HD, HP-HD and conservative therapy for $\mathrm{PQ}$ poisoning in one trial; hence, data from different studies are adopted for sample size calculation. Studies of bigger sample size and those that have a similar design to our research are preferentially selected for reference. Gao et al compared HP $(\mathrm{n}=458)$ and HP+Continuous Veno-Venous Hemofiltration $(\mathrm{CVVH}) \quad(\mathrm{n}=226)$ in $\mathrm{PQ}$ poisoning treatment, and reported that the mortality of HP was 57.4\%. ${ }^{19}$ Park and colleagues investigated the efficacy of HP-HD consecutive therapy $(n=347)$ and concurrent therapy $(n=383)$ and found that HP-HD concurrent therapy had a lower mortality (57.9\% vs $81.8 \%) .{ }^{28}$ In a Chinese study by Liu et al, the mortality of conservative therapy for PQ poisoned patients was $78.2 \%(\mathrm{n}=87) .{ }^{29}$ Even less evidence could be obtained in HD treatment for PQ poisoning in the last 30 years. Proudfoot $e t$ al investigated the efficacy of HD in clearing $\mathrm{PQ}$, but since both $\mathrm{HD}$ and peritoneal dialysis were included in the active arm, ${ }^{30}$ it is not considered for sample size estimation. Eventually a Chinese study by Yang ${ }^{31}$ is adopted, and they concluded that mortality of $\mathrm{HD}$ was as low as $38.10 \%$ ( $\mathrm{n}=26)$, as compared with $88.24 \%$ in the control group $(\mathrm{n}=17)$. Though the absolute sample size was small, it is the largest that we could find, and the investigated intervention did not include peritoneal dialysis; thus, it is selected for reference.

With the 28-day mortality being the primary outcome, and $\mathrm{p}<0.05$ defined as significantly different, the $\mathrm{Z}$ test with pooled variance ${ }^{32-36}$ is applied to calculate the sample size (study power $80 \%$ ). Based on these data, at least 78, 13 and 81 subjects would be needed for HP, HD and HP-HD group, respectively. As the subjects in each group is set at a 1:1:1:1 ratio, a sample size of 81 per group is adopted. With an estimated dropout rate of $10 \%$, 90 patients would be enrolled for each group eventually.

\section{Monitoring}

Arterial blood gas test, complete blood count, coagulation function test, liver function and pancreatic function would be performed and urine volume would be taken every day before haemopurification (if there is any). Urine dithionite test result would be recorded every 4-6hours from admission until there are three consecutive negative results. Renal function would be tested daily. ${ }^{10}$ Chest radiographs would be taken once a week or as soon as the patient deteriorates. If any patient develops fever or sepsis during treatment, they would be investigated to identify potential catheter-related bloodstream infection. Ultrasound for lower limb deep veins would be administered for patients with notable increase of calf/ thigh circumference to identify thrombogenesis.

\section{Outcomes}

Twenty-eight-day mortality would be the primary endpoint for this trial, which is a commonly used measurement ${ }^{1928293137}$ as most death events occur during this period. ${ }^{11}$ The result would be presented in terms of percentage and $95 \% \mathrm{CI}$.

Secondary outcomes include: (1) survival time (from the time of $\mathrm{PQ}$ ingestion to the time of death), all-cause mortality at the 3rd, 7th and 60th days; (2) rate of necessary oxygen uptake and rate of mechanical ventilation; (3) in-hospital length of stay and ICU length of stay; (4) APACHE II score and PSS score; (5) rate of general complications, such as respiratory failure, acute kidney injury (AKI), acute liver failure, pancreas function abnormality and multiple organ failure (MOF); (6) rate of intervention-related complications, such as catheter placement-related complications, thrombocytopenia and deep venous thrombosis; (7) rate of adverse events, which include unexpected death, severe haemorrhage or oedema, unplanned extubation, coagulation in the extracorporeal circulation, blockage of cartridge, incorrect pipe connection, etc. These results would be presented in the form of mean value and $95 \%$ CI. (4) would be assessed at admission. (2), (3), (5), (6) and (7) would be recorded during hospitalisation and reviewed by the time of discharge or in-hospital death. Death events would be recorded during hospitalisation. Patients who are discharged would receive a followed-up phone call at the 60 th day from PQ intoxication. All death events would be recorded by date to calculate survival time and mortality at 3rd, 7th, 28th and 60th days. For patients who discontinue or change therapy, data would be collected at the termination of assigned treatment.

\section{Patient involvement}

No patients were involved in the development of the research questions or the outcome measures, nor were they invited to develop the plans for design, recruitment or conduction of the study. No patients were asked to assess the burden of intervention. The result will not be disseminated to participants or the relevant communities.

\section{Participant timeline}

The study would start after the manuscript is accepted, and it is expected to be completed in 3 years or more depending on actual enrolment. The timeline of participant is listed in table 3 .

\section{Data collection and management}

All participants would be given a study ID, and all information would be saved by study ID in an electronic 
Table 3 Participant timeline

\begin{tabular}{llll} 
& Enrolment & $\begin{array}{l}\text { Discharge from } \\
\text { hospital }\end{array}$ & Day 60 \\
\hline $\begin{array}{l}\text { Check the } \\
\text { inclusion and } \\
\text { exclusion criteria }\end{array}$ & $\sqrt{ }$ & \\
$\begin{array}{l}\text { Sign informed } \\
\text { consents }\end{array}$ & $\sqrt{ }$ & \\
$\begin{array}{l}\text { Allocation and } \\
\text { intervention }\end{array}$ & $\sqrt{ }$ & & \\
$\begin{array}{l}\text { Assessment } \\
\text { Report and fill the } \\
\text { case report forms }\end{array}$ & & $\sqrt{ }$ \\
$\begin{array}{l}\text { Survival status } \\
\text { Follow-up }\end{array}$ & $\sqrt{ }$ & \\
\hline
\end{tabular}

database. All data in this trial would be recorded and saved as electronic case report form (eCRF), kept and managed by the Emergency Department of Peking Union Medical College Hospital. There would be two databases containing information of this trial, one of which (database 1) only contains information of the ID number, name and intervention of each participant, while another (database 2) contains the ID number, grouping information and clinical data of the patient without intervention details. Statisticians only have access to database 2. Frontline physicians would have restricted access only to the data of the patients that they are directly involved with. Database 1 would be managed by an independent person who has no interest of conflict in this study. All of the envelopes given to physicians with assignment information would be preserved and kept in a locker by the chief data manager. All clinical data including adverse events collected during hospitalisation can be obtained from electronic medical record system or paper notes. Contact information of patients and their family members would be required on admission. Information on patient deaths can be obtained from medical records and follow-up calls.

\section{Statistical analysis}

Considering the high cost of each participant, intentionto-treat (ITT) analysis would be adopted to fully use the data. Dropout rate, which may increase the bias of ITT analysis, would stay low in this trial with the relatively short course of disease. To obtain a relatively conservative result, the last observation carried forward method would be used to fill up missing and dropout data. The missing data of survival would be carried forward as death, so as to reduce potential treatment effect bias induced by the active arms. Results would be calculated by SAS V.9.3, and $\mathrm{p}<0.05$ is defined as statistically significant. The Cox regression model (5\% significance level) would be applied to examine the relationship between 28-day mortality and intervention group, $\mathrm{PQ}$ ingestion amount, urine dithionite test results, time lapse from intoxication to treatment, age and the acid-base or electrolyte status on admission. For secondary outcome (2), (5), (6) and (7), RxC contingency tables would be used to test the difference of these indicators in four groups. If significant differences are found, Bonferroni test would be performed to find treatment effect differences between each group. As for length of stay and scores, one-way analysis of variance would be applied. Exploratory subgroup analysis would be performed to investigate treatment effect in different patients. Patients would be divided into subgroups by these factors: urine dithionite test result (light blue, navy blue and dark blue), and time from ingestion to treatment ( $\geq 4$ hours and $<4$ hours). The survival time of each group would also be analysed with the help of log-rank test, Cox regression and KaplanMeier survival curve.

\section{Data monitoring}

The data monitoring committee (DMC) consists of three independent physicians and one statistician. It is responsible for regular review of accumulating trial data on efficacy and safety. It can also suggest to trial sponsor and investigator on trial continuation, modification or cessation based on benefit-risk assessment. Every 4 months, the DMC would hold a meeting to review statistical reports presented by Statistical Data Analysis Center, which is composed of a group of statisticians. The DMC would have access to unmasked results on 28-day mortality, survival time, rate of MOF and rate of severe complications. These outcomes would be kept confidential by DMC unless a clear difference is observed among groups and DMC requests trial termination. It would also review the occurrence of serious adverse events, which include unexpected death, severe haemorrhage or oedema, etc. Adverse events would be collected by self-report by physicians and nurses in charge of the subjects on the eCRF system. The DMC would evaluate these events in the meetings and decide if an early end to the trial should be applied. Inter-rater agreement would be assessed by $\kappa$ analysis.

\section{Definitions}

CKD is defined according to Kidney Disease Outcomes Quality Initiative Guideline as damage or decrease of kidney function, ${ }^{38}$ which presents as urinary albumin excretion $\geq 30 \mathrm{mg}$ /day or estimated glomerular filtration rate $(\mathrm{eGFR}) \leq 60 \mathrm{~mL} / \mathrm{min} / 1.73 \mathrm{~m}^{2}$ for 3 months or more.

According to Kidney Disease Improving Global Outcomes classification, ${ }^{39}$ AKI is diagnosed in patients who meet any criteria of the following: (1) Increase in serum creatinine $\geq 0.3 \mathrm{mg} / \mathrm{dL}$ in 48 hours. (2) The serum creatinine has increased to more than 1.5 times than baseline within 7 days. (3) The volume of urine is lease than $0.5 \mathrm{~mL} / \mathrm{kg} /$ hour for 6 hours.

COPD is defined according to the Global Initiative for Chronic Obstructive Lung Disease criteria. ${ }^{40}$ Patients whose spirometry result indicates air flow limitation (forced expiratory volume in $1 \mathrm{~s} /$ forced vital capacity $<0.7)$ after bronchodilator inhalation without alternative 
explanation for patients' symptoms can be diagnosed as COPD.

Respiratory failure can be diagnosed in the patients with an arterial oxygen $\mathrm{PaO}_{2}<60 \mathrm{~mm} \mathrm{Hg}$ in air pressure of sea level, with or without $\mathrm{PaCO}_{2}>50 \mathrm{~mm} \mathrm{Hg}$.

Chronic liver disease is defined as disease of the liver lasting longer than 6 months. Cirrhosis, chronic liver inflammation caused by infection or autoimmune disease are included in chronic liver disease. Cirrhosis is defined according the National Institute for Health and Care Excellence 2016 guideline, ${ }^{41}$ in which patients can be diagnosed with cirrhosis with typical imaging, laboratory results together with risk factors or with biopsy confirmation alone.

Acute liver failure is defined as acute damage in liver function without obvious history of liver disease or cirrhosis within 26 weeks. Patients who meet all the following criteria can be diagnosed as acute liver failure ${ }^{42}$ : elevated aminotransferases, mental alteration (hepatic encephalopathy) and INR (international normalised ratio) $\geq 1.5$.

ACS is associated with myocardial ischaemia, which includes ST elevation myocardial infarction (STEMI), unstable angina (UA) and non-STEMI (NSTEMI).

ARDS is defined according to Berlin definition. ${ }^{43}$ Patients who meet all the criteria below can be diagnosed with ARDS: (1) The respiratory symptoms must occur within 1 week of noticed clinical disease, or patients' present new symptoms or respiratory symptoms in 1 week. (2) Chest X- ray or CT shows signs of pneumonoedema in both sides of lungs which cannot be fully explained by pleural effusion, atelectasis, lobe collapse or pulmonary nodules. (3) Heart failure and fluid overload cannot completely explain the respiratory failure. (4) The patient must present with moderate to severe oxygen impairment which can be defined by the ratio of $\mathrm{PaO}_{2} / \mathrm{FiO}_{2}$. When the positive end-expiratory pressure (PEEP) is set as $5 \mathrm{cmH}_{2} \mathrm{O}$ or more, the $\mathrm{PaO}_{2} / \mathrm{FiO}_{2}$ is less than $300 \mathrm{~mm} \mathrm{Hg}$.

Abnormal pancreatic function is defined as serum amylase $>220 \mathrm{U} / \mathrm{L}$, which can be classified into two degrees, mildly elevated (220-660 U/L) and elevated $(>660 \mathrm{U} / \mathrm{L}) .^{20}$

Multiorgan dysfunction is defined according to the Sepsis-3 definition: patients with Sequential Organ Failure Assessment (SOFA) score $\geq 2$ are determined to have multiorgan dysfunction or MOF. ${ }^{44}$

\section{Ethics and dissemination}

If important modifications or decision are made, the Ethics Committee of the First Affiliated Hospital of Zhengzhou University would be informed, and new protocols would be uploaded to Clinicaltrials.gov.

All eligible participants and their family members would be given informed consent documents with adequate time to consider and communicate with physicians. Consent provisions for collection and use of participant data and biological specimens in potential ancillary studies are also included in the informed consent. Refusal to participate in this trial would not influence the care they receive under any circumstances. Discontinuation or modification of treatment could be requested by patients and their families, or in cases of allergic responses to haemopurification materials. Serious events and unexpected adverse events would be recorded and reported to the Ethics Committee of the First Affiliated Hospital of Zhengzhou University and DMC. An independent audit would be held every 6 months to supervise trial conduct. Three toxicologists and three independent statisticians would be invited to the audit. Personal contact information would be accessible only to the research team members who are in charge of follow-up. Full protocol would be accessible to the public on BMJ Open. The results of the present study would be published in international peer-reviewed journals. Original research data could be requested from the corresponding author.

Contributors $\mathrm{YL}, \mathrm{Y}-\mathrm{XG}, \mathrm{XY}$ and $\mathrm{HZ}$ raised the idea and developed the plan for the trial. YL and Y-XG formulated the intervention plan. J-WC and YX wrote the original paper. J-WC, YX, XL, SY and YM collected the reference data for the trial and designed the case report form (CRF). Y-XG, YW, DY, LC and PS prepared the documents for ethical review and obtained the research ethical approvals. SG revised the paper and work in English. MW reviewed the reference data, calculated the sample size and helped design the analysis plan. YL reviewed and embellished the original paper, and confirmed the final protocol. All authors reviewed the final version of manuscript.

Funding This study is supported by the National Natural Science Foundation of China, grant number \#81701893 (Y-XG) and 81550034 (YL). The sponsor of this trial was not involved in the study design, data collection, result analysis, writing of the report or publication.

Competing interests None declared.

Patient consent Obtained.

Ethics approval The Ethics Committee of The First Affiliated Hospital of Zhengzhou University in September 2017 (2017-KY-10).

Provenance and peer review Not commissioned; externally peer reviewed.

Open access This is an open access article distributed in accordance with the Creative Commons Attribution Non Commercial (CC BY-NC 4.0) license, which permits others to distribute, remix, adapt, build upon this work non-commercially, and license their derivative works on different terms, provided the original work is properly cited and the use is non-commercial. See: http://creativecommons.org/ licenses/by-nc/4.0/

(c) Article author(s) (or their employer(s) unless otherwise stated in the text of the article) 2018. All rights reserved. No commercial use is permitted unless otherwise expressly granted.

\section{REFERENCES}

1. Eddleston M, Phillips MR. Self poisoning with pesticides. BMJ 2004;328:42-4.

2. Bertolote JM, Fleischmann A, Eddleston M, et al. Deaths from pesticide poisoning: a global response. $B r J$ Psychiatry 2006;189:201-3.

3. Gawarammana IB, Buckley NA. Medical management of paraquat ingestion. Br J Clin Pharmacol 2011;72:745-57.

4. Gil HW, Kang MS, Yang JO, et al. Association between plasma paraquat level and outcome of paraquat poisoning in 375 paraquat poisoning patients. Clin Toxicol 2008;46:515-8.

5. Senarathna L, Eddleston M, Wilks MF, et al. Prediction of outcome after paraquat poisoning by measurement of the plasma paraquat concentration. QJM 2009;102:251-9.

6. Hong SY, Lee JS, Sun IO, et al. Prediction of patient survival in cases of acute paraquat poisoning. PLoS One 2014;9:e111674.

7. Dawson AH, Eddleston M, Senarathna L, et al. Acute human lethal toxicity of agricultural pesticides: a prospective cohort study. PLOS Med 2010;7:e1000357. 
8. Lin JL, Leu ML, Liu YC, et al. A prospective clinical trial of pulse therapy with glucocorticoid and cyclophosphamide in moderate to severe paraquat-poisoned patients. Am J Respir Crit Care Med 1999:159:357-60.

9. Lin JL, Lin-Tan DT, Chen KH, et al. Repeated pulse of methylprednisolone and cyclophosphamide with continuous dexamethasone therapy for patients with severe paraquat poisoning. Crit Care Med 2006;34:368-73

10. Lin JL, Lin-Tan DT, Chen KH, et al. Improved survival in severe paraquat poisoning with repeated pulse therapy of cyclophosphamide and steroids. Intensive Care Med 2011;37:1006-13.

11. section CMDAep. Expert advice on diagnosis and treatment of acute paraquat poisoning in China(2013) [in Chinese]. Chin J Crit Care Med 2013;33:484-9.

12. Hong SY, Yang JO, Lee EY, et al. Effect of haemoperfusion on plasma paraquat concentration in vitro and in vivo. Toxicol Ind Health 2003;19:17-23.

13. Wunnapuk K, Mohammed F, Gawarammana I, et al. Prediction of paraquat exposure and toxicity in clinically ill poisoned patients: a model based approach. Br J Clin Pharmacol 2014;78:855-66.

14. Ghannoum M, Nolin TD, Lavergne V, et al. Blood purification in toxicology: nephrologys ugly duckling. Adv Chronic Kidney Dis 2011;18:160-6.

15. Hedaiaty M, Sabzghabaee A, Gheshlaghi F, et al. Paraquat poisoning management in iran (isfahan): devising a protocol. Br J Med Med Res 2016;16:1-10.

16. Kang MS, Gil HW, Yang JO, et al. Comparison between kidney and hemoperfusion for paraquat elimination. $J$ Korean $\mathrm{Med} \mathrm{Sci}$ 2009;24(Suppl):S156-60.

17. Li A, Li W, Hao F, et al. Early stage blood purification for paraquat poisoning: a multicenter retrospective study. Blood Purif 2016;42:93-9.

18. Rao R, Bhat R, Pathadka S, et al. Golden hours in severe paraquat poisoning-the role of early haemoperfusion therapy. J Clin Diagn Res 2017;11-Oc06-oc08.

19. Gao $Y$, Zhang $X$, Yang $Y$, et al. Early haemoperfusion with continuous venovenous haemofiltration improves survival of acute paraquatpoisoned patients. J Int Med Res 2015;43:26-32.

20. Li GQ, Li YM, Wei LQ, et al. Comparison between kidney and continuous plasma perfusion for paraquat elimination. Am J Med Sci 2014;348:195-203.

21. Cao Y, Yang X, Li T. Effect of hemoperfusion rate on prognosis of patients with acute paraquat poisoning [in Chinese]. Chin Crit Care Med 2016;28:870-5.

22. Cao Q, Qian Y, Li J, et al. Analysis of 43 cases of acute paraquat poisoning treated by concurrent hemoperfusion and hemodialysis treatment [in Chinese]. Guide China Med 2011;09:219-20.

23. Deng C, Xiong J, Wang T, et al. Effects of hemoperfusion on paraquat plasma concentration and prognosis of paraquat poisoning patients [in Chinese]. Chin J Crit Care Med 2005;25:928-9.
24. Xue L, Chen M, Liu N, et al. Efficacy of hemoperfusion combined with hemodialysis in the treatment of paraquat poisoning [in Chinese]. Chin J Blood Purif 2014;02:82-4.

25. Yadong Z, Jixue S, Ling Y, et al. Comparative study of difierent methods of blood purification treatment of paraquat intoxication. Chin J Ind Hyg Occup Dis 2014;32:862-4.

26. Cheng X, Ding X, Liu F, et al. Standard practice for blood purification in China: 2010 edition [in Chinese]. 2010.

27. Nilsson $\mathrm{H}$, Ekberg $\mathrm{O}$, Olsson R, et al. Quantitative assessment of swallowing in healthy adults. Dysphagia 1996;11:110-6.

28. Park S, Lee S, Park S, et al. Concurrent hemoperfusion and hemodialysis in patients with acute pesticide intoxication. Blood Purif 2016;42:329-36.

29. Xiao-wei L, Ye-sheng L, Zhi L. Analysis of clinic efficacy on early repeated hemoperfusion in patients with acute paraquat poisoning. Chin J Emerg Med 2012;21:1205-9.

30. Proudfoot AT, Prescott LF, Jarvie DR. Haemodialysis for paraquat poisoning. Hum Toxicol 1987;6:69-74.

31. Yang Y. Acute paraquat poisoning rescue experience. Anhui Med $J$ 1999;20:54-5.

32. D'agostino RB, Chase W, Belanger A. The appropriateness of some common procedures for testing the equality of two independent binomial populations. The American Statistician 1988;42:198-202.

33. Chow S-C, Wang H, Shao J. Sample size calculations in clinical research. US: CRC press, 2007.

34. Lachin JM. Biostatistical methods: the assessment of relative risks. US: John Wiley \& Sons, 2009.

35. Machin D, Campbell MJ, Tan S-B, et al. Sample size tables for clinical studies. US: John Wiley \& Sons, 2011.

36. Fleiss JL, Levin B, Paik MC. Statistical methods for rates and proportions. US: John Wiley \& Sons, 2013.

37. Jin K, Guo L, Shao M, et al. [Intensity of hemoperfusion in acute paraquat-poisoned patients and analysis of prognosis]. Zhonghua Wei Zhong Bing Ji Jiu Yi Xue 2015;27:263-9.

38. National Kidney Foundation. K/DOQI clinical practice guidelines for chronic kidney disease: evaluation, classification, and stratification. Am J Kidney Dis 2002;39(2 Suppl 1):S1.

39. Khwaja A. KDIGO clinical practice guidelines for acute kidney injury. Nephron Clin Pract 2012;120:c179-c184.

40. Fabbri LM, Hurd SS. GOLD Scientific Committee. Global strategy for the diagnosis, management and prevention of COPD: 2003 update. Eur Respir J 2003;22:1.

41. Guideline N. Cirrhosis in over 16s: assessment and management. 2016.

42. Polson J, Lee WM. American Association for the Study of Liver Disease. AASLD position paper: the management of acute liver failure. Hepatology 2005;41:1179-97.

43. Force ADT. Acute respiratory distress syndrome. Jama 2012;307:2526-33.

44. Singer M, Deutschman CS, Seymour CW, et al. The Third International Consensus definitions for sepsis and septic shock (Sepsis-3). JAMA 2016;315:801-10. 